\begin{tabular}{|c|c|}
\hline Title & $\begin{array}{l}\text { A vector finite element method with the high-order mixed-interpolation-type triangular elements for optical } \\
\text { waveguiding problems }\end{array}$ \\
\hline Author(s) & Koshiba, M.; Maruyama, S.; Hirayama, K. \\
\hline Citation & $\begin{array}{l}\text { Journal of Lightwave Technology, 12(3), 495-502 } \\
\text { https://doi.org/10.1109/50.285332 }\end{array}$ \\
\hline Issue Date & $1994-03$ \\
\hline Doc URL & http:/hdl.handle.net/2115/6038 \\
\hline Rights & $\begin{array}{l}\text { C1994 IEEE. Personal use of this material is permitted. However, permission to reprint/republish this material for } \\
\text { advertising or promotional purposes or for creating new collective works for resale or redistribution to servers or lists, } \\
\text { or to reuse any copyrighted component of this work in other works must be obtained from the IEEE." } \\
\text { IEEE, Journal of Lightwave Technology, 12(3), 1994, p495-502 }\end{array}$ \\
\hline Type & article \\
\hline File Information & JLT12_3.pdf \\
\hline
\end{tabular}

Instructions for use 


\title{
A Vector Finite Element Method With the High-Order Mixed-Interpolation-Type Triangular Elements for Optical Waveguiding Problems
}

\author{
Masanori Koshiba, Senior Member, IEEE, Shinji Maruyama, and Koichi Hirayama, Member, IEEE
}

\begin{abstract}
A vector finite element method with the high-order mixed-interpolation-type triangular elements is described for the analysis of optical waveguiding problems. It is a combination of linear edge elements for transverse components of the electric or magnetic field and quadratic nodal elements for the axial one. The use of mixed-interpolation-type elements provides a direct solution for propagation constants and avoids spurious solutions. This approach can yield more accurate results compared with the conventional approach using the lowest order mixedinterpolation-type elements, namely, constant edge elements and linear nodal elements. The accuracy of this approach is investigated by calculating the propagation characteristics of optical rib waveguides. Results obtained for both $E^{x}$ and $E^{y}$ polarizations are validated using benchmark results produced by established methods.
\end{abstract}

\section{INTRODUCTION}

D IFFERENT types of the vector finite element method (VFEM) have been developed for the analysis of optical waveguiding problems. Of the various formulations, the VFEM using full vector electric or magnetic field is quite suitable for a wide range of practical complicated problems [1]-[13]. This approach has been widely used for various optical waveguiding structures and recently has been utilized as the optical waveguide solver of CAD packages [14]. The most serious problem associated with this approach is the appearance of spurious solutions. The penalty function method [1]-[14] has been used to cure this problem, but in this technique an arbitrary positive constant, called the penalty coefficient, is involved and the accuracy of solutions depends on its magnitude. Furthermore, in the full vectorial formulation the propagation constant is first given as an input datum, and subsequently the operating wavelength is obtained as a solution. There is another serious problem in the full vectorial approach. As was made clear by Birman [15] and Birman and Solomyak [16], such an approach is quite difficult for dealing with corner singularities and interface singularities so long as the conventional Lagrange interpolation polyno-

Manuscript received November 25, 1992; revised February 22, 1993. This work was supported in part by a Grant-in-Aid for Scientific Research on Priority Areas, Ultrafast and Ultra-Parallel Optoelectronics from the Ministry of Education, Science, and Culture, Japanese Government.

M. Koshiba and S. Maruyama are with the Department of Electronic Engineering, Hokkaido University, Sapporo, 060 Japan.

K. Hirayama is with the Department of Electronic Engineering, Kitami Institute of Technology, Kitami, 090 Japan.

IEEE Log Number 9210178. mial functions are used to approximate vector fields. More recently, the VFEM with the lowest order mixed-interpolationtype triangular elements, namely, constant edge elements for transverse components of the electric or magnetic field and linear nodal (conventional Lagrange [1]-[14]) elements for the axial one, has been developed [17]-[19]. The use of mixed-interpolation-type elements provides a direct solution for propagation constants [18] and avoids spurious solutions [17]-[19], but the accuracy of the finite element analysis using the lowest order elements is, in general, insufficient.

In this paper, in order to provide more accurate numerical solutions and faster convergence in applications, a vector finite element method with the high-order mixed-interpolation-type triangular elements is formulated in detail. It is a combination of linear edge elements for transverse components of the electric or magnetic field and quadratic nodal (conventional Lagrange) elements for the axial one. This approach can yield more accurate results compared with the conventional approach using the lowest order elements. The accuracy of this approach is investigated by calculating the propagation characteristics of optical rib waveguides. Results obtained for both $E^{x}$ and $E^{y}$ polarizations are validated using benchmark results produced by established methods.

\section{BASIC EQUATIONS}

We consider an optical waveguide with an arbitrary cross section $\Omega$ in the $x y$ plane. With a time dependence of the form $\exp (j \omega t)$ being implied, from Maxwell's equations the following vectorial wave equation is derived:

$$
\nabla \times([p] \nabla \times \phi)-k_{0}^{2}[q] \phi=0
$$

with

$$
\begin{aligned}
& {[p]=\left[\begin{array}{ccc}
p_{x} & 0 & 0 \\
0 & p_{y} & 0 \\
0 & 0 & p_{z}
\end{array}\right]} \\
& {[q]=\left[\begin{array}{ccc}
q_{x} & 0 & 0 \\
0 & q_{y} & 0 \\
0 & 0 & q_{z}
\end{array}\right]}
\end{aligned}
$$

where $\omega$ is the angular frequency, $k_{0}$ is the free-space wavenumber, $\phi$ denotes either the electric field $E$ or the magnetic field $\boldsymbol{H}$, and the components of $[p]$ and $[q]$ are given 


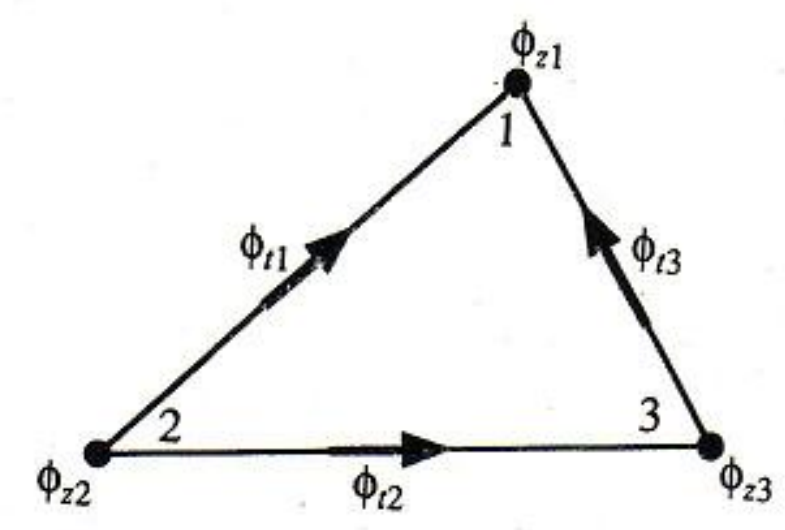

(a)

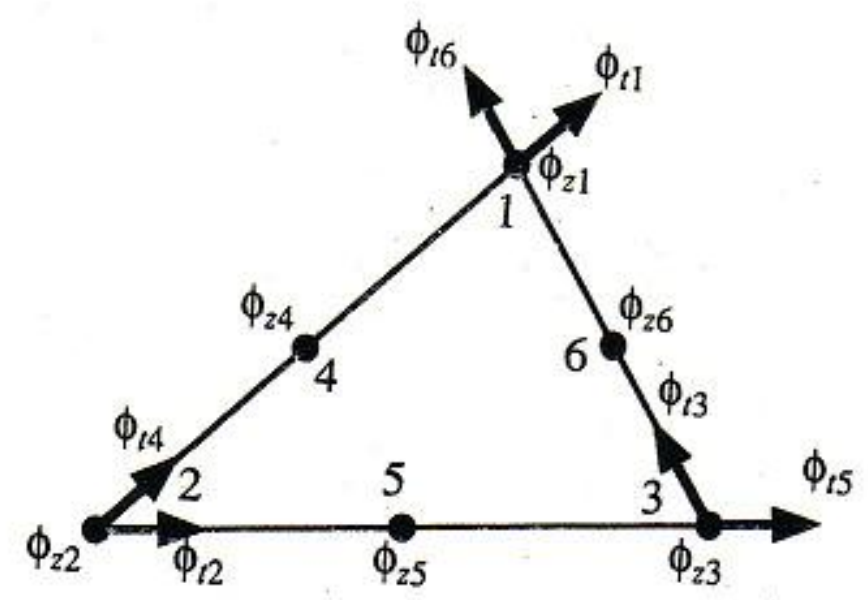

(b)

Fig. 1. Mixed-interpolation-type triangular element. (a) Constant edge and linear nodal elements. (b) Linear edge and quadratic nodal elements.

by

$$
\begin{aligned}
p_{x} & =p_{y}=p_{z}=1 \\
q_{x} & =n_{x}^{2} \\
q_{y} & =n_{y}^{2} \\
q_{z} & =n_{z}^{2}, \quad \text { for } \phi=\boldsymbol{E} \\
p_{x} & =1 / n_{x}^{2} \\
p_{y} & =1 / n_{y}^{2} \\
p_{z} & =1 / n_{z}^{2} \\
q_{x} & =q_{y}=q_{z}=1, \quad \text { for } \phi=\boldsymbol{H} .
\end{aligned}
$$

Here $n_{x}, n_{y}, n_{z}$ are the refractive indices in the $x, y, z$ directions, respectively.

The functional for (1) is given by

$$
F=\iint_{\Omega}\left[(\nabla \times \phi)^{*} \cdot([p] \nabla \times \phi)-k_{0}^{2}[q] \phi^{*} \cdot \phi\right] d x d y
$$

where the asterisk denotes complex conjugate.

\section{MIXED-INTERPOLATION-TYPe TRIANGULAR ElEMENTS}

The electromagnetic fields have to be tangentially continuous across material interfaces.

Fig. 1(a) shows the lowest order mixed-interpolation-type triangular element [17]-[19] which is composed of a constant edge element with three tangential unknowns, $\phi_{t 1}$ to $\phi_{t 3}$, and a linear nodal (conventional Lagrange) element with three axial unknowns, $\phi_{z 1}$ to $\phi_{z 3}$. Since both $\phi_{t}$ and $\phi_{z}$ are tangential to material interfaces, the tangential continuity can be straight forwardly imposed in the mixed-interpolation-type element analysis. In this lowest order element the tangential component $\phi_{t}=\phi \cdot t$ is constant along each side of triangles, where $t$ is the unit tangential vector whose direction is coincident with that of $\phi_{t}$, as shown in Fig. 1(a). It is for this reason that the edge element in Fig. 1(a) is called the constant edge element.
TABLE I

SHAPE FUNCTION VECTORS

\begin{tabular}{cccc}
\hline \hline Elements & $\{U\}$ & $\{V\}$ & $\{N\}$ \\
\hline $\begin{array}{c}\text { Constant edge } \\
\text { and linear } \\
\text { nodal elements }\end{array}$ & $\frac{1}{2 A_{e}}\left[\begin{array}{l}l_{1}\left(y_{3}-y\right) \\
l_{2}\left(y_{1}-y\right) \\
l_{3}\left(y_{2}-y\right)\end{array}\right]$ & $\frac{1}{2 A_{e}}\left[\begin{array}{l}l_{1}\left(x-x_{3}\right) \\
l_{2}\left(x-x_{1}\right) \\
l_{3}\left(x-x_{2}\right)\end{array}\right]$ & {$\left[\begin{array}{l}L_{1} \\
L_{2} \\
L_{3}\end{array}\right]$} \\
\hline $\begin{array}{c}\text { Linear edge } \\
\text { and quadratic } \\
\text { nodal elements }\end{array}$ & $\frac{1}{2 A_{e}}\left[\begin{array}{r}l_{1} b_{2} L_{1} \\
l_{2} b_{3} L_{2} \\
l_{3} b_{1} L_{3} \\
-l_{1} b_{1} L_{2} \\
-l_{2} b_{2} L_{3} \\
-l_{3} b_{3} L_{1}\end{array}\right]$ & $\frac{1}{2 A_{e}}\left[\begin{array}{c}l_{1} c_{2} L_{1} \\
l_{2} c_{3} L_{2} \\
l_{3} c_{1} L_{3} \\
-l_{1} c_{1} L_{2} \\
-l_{2} c_{2} L_{3} \\
-l_{3} c_{3} L_{1}\end{array}\right]\left[\begin{array}{c}L_{1}\left(2 L_{1}-1\right) \\
L_{2}\left(2 L_{2}-1\right) \\
L_{3}\left(2 L_{3}-1\right) \\
4 L_{1} L_{2} \\
4 L_{2} L_{3} \\
4 L_{3} L_{1}\end{array}\right]$ \\
\hline
\end{tabular}

Fig. 1(b) shows the high-order mixed-interpolation-type triangular element which is composed of a linear edge element with six tangential unknowns defined at the three vertices of the triangle, $\phi_{t 1}$ to $\phi_{t 6}$, and a quadratic nodal (conventional Lagrange) element with six axial unknowns, $\phi_{z 1}$ to $\phi_{z 6}$. In this high-order element which, to our knowledge, has not been utilized so far, the tangential component $\phi_{t}$ along each side of triangles is approximated to linear order. Hano [20] used a linear edge element with six tangential unknowns defined at the six nodal points within each element. This requires the users to select a suitable location for the nodal points. Lee et al. [21] proposed using the second-order Lagrange interpolation polynomial. This approach requires two facial unknowns in addition to six edge variables to provide a quadratic approximation of the normal component of the field along any two of the three sides of the triangle. The linear edge element was previously introduced by Brezzi et al. [22] and Durán [23] for two-dimensional problems, and by Nédélec [24] for three-dimensional problems. Its explicit form of shape functions, however, is not given there.

\section{FINITE ELEMENT DISCRETIZATION}

Dividing the waveguide cross section $\Omega$ into a number of mixed-interpolation-type triangular elements, as shown in Fig. 1 , we expand the transverse components $\phi_{x}, \phi_{y}$ and the axial component $\phi_{z}$ in each element as

$$
\phi=\left[\begin{array}{c}
\phi_{x} \\
\phi_{y} \\
\phi_{z}
\end{array}\right]=\left[\begin{array}{r}
\{U\}^{\mathrm{T}}\left\{\phi_{t}\right\}_{e} \\
\{V\}^{\mathrm{T}}\left\{\phi_{t}\right\}_{e} \\
j\{N\}^{\mathrm{T}}\left\{\phi_{z}\right\}_{e}
\end{array}\right]
$$

where $\left\{\phi_{t}\right\}_{e}$ is the edge variables in the transverse plane for each element, $\left\{\phi_{z}\right\}_{e}$ is the nodal axial-field vector for each element, and $\mathrm{T}$ denotes a transpose. The shape function vectors for edge elements $\{U\}$ and $\{V\}$ and the ordinary shape function vector for nodal elements $\{N\}$ are given in Table I, where the area coordinates $L_{k}(k=1,2,3)$, the area of the element $A_{e}$, the length of the side between two corner points $\left(x_{k}, y_{k}\right)$ and $\left(x_{l}, y_{l}\right),\left|l_{k}\right|$, and coefficients $a_{k}, b_{k}, c_{k}$ are given by

$$
\left[\begin{array}{l}
L_{1} \\
L_{2} \\
L_{3}
\end{array}\right]=\frac{1}{2 A_{e}}\left[\begin{array}{lll}
a_{1} & b_{1} & c_{1} \\
a_{2} & b_{2} & c_{2} \\
a_{3} & b_{3} & c_{3}
\end{array}\right]\left[\begin{array}{l}
1 \\
x \\
y
\end{array}\right]
$$


TABLE II

DERIVATIVES OF SHAPE FunCTIONS

\begin{tabular}{ccccc}
\hline \hline Elements & $\left\{U_{y}\right\}$ & $\left\{V_{x}\right\}$ & $\left\{N_{x}\right\}$ & $\left\{N_{y}\right\}$ \\
\hline $\begin{array}{c}\text { Constant edge } \\
\text { and linear } \\
\text { nodal elements }\end{array}$ & $\frac{1}{2 A_{e}}\left[\begin{array}{l}-l_{1} \\
-l_{2} \\
-l_{3}\end{array}\right]$ & $\frac{1}{2 A_{e}}\left[\begin{array}{l}l_{1} \\
l_{2} \\
l_{3}\end{array}\right]$ & $\frac{1}{2 A_{e}}\left[\begin{array}{l}b_{1} \\
b_{2} \\
b_{3}\end{array}\right]$ & $\frac{1}{2 A_{e}}\left[\begin{array}{l}c_{1} \\
c_{2} \\
c_{3}\end{array}\right]$ \\
\hline $\begin{array}{c}\text { Linear edge } \\
\text { nod quadratic elements }\end{array}$ & $\frac{1}{4 A_{e}^{2}}\left[\begin{array}{r}l_{2} b_{3} c_{2} \\
l_{3} b_{1} c_{3} \\
-l_{1} b_{1} c_{2} \\
-l_{2} b_{2} c_{3} \\
-l_{3} b_{3} c_{1}\end{array}\right] \frac{1}{4 A_{e}^{2}}\left[\begin{array}{c}l_{1} c_{2} b_{1} \\
l_{2} c_{3} b_{2} \\
l_{3} c_{1} b_{3} \\
-l_{1} c_{1} b_{2} \\
-l_{2} c_{2} b_{3} \\
-l_{3} c_{3} b_{1}\end{array}\right] \frac{1}{2 A_{e}}\left[\begin{array}{c}b_{1}\left(4 L_{1}-1\right) \\
b_{2}\left(4 L_{2}-1\right) \\
b_{3}\left(4 L_{3}-1\right) \\
4\left(b_{1} L_{2}+b_{2} L_{1}\right) \\
4\left(b_{2} L_{3}+b_{3} L_{2}\right) \\
4\left(b_{3} L_{1}+b_{1} L_{3}\right)\end{array}\right] \frac{1}{2 A_{e}}\left[\begin{array}{c}c_{1}\left(4 L_{1}-1\right) \\
c_{2}\left(4 L_{2}-1\right) \\
c_{3}\left(4 L_{3}-1\right) \\
4\left(c_{1} L_{2}+c_{2} L_{1}\right) \\
4\left(c_{2} L_{3}+c_{3} L_{2}\right) \\
4\left(c_{3} L_{1}+c_{1} L_{3}\right)\end{array}\right]$ \\
\hline
\end{tabular}

$2 A_{e}=\left|\begin{array}{ccc}1 & 1 & 1 \\ x_{1} & x_{2} & x_{3} \\ y_{1} & y_{2} & y_{3}\end{array}\right|$

$l_{k}=\left\{\begin{aligned} \sqrt{b_{m}^{2}+c_{m}^{2}}, & \text { for } b_{m}<0 \text { or } b_{m}=0, c_{m}>0 \\ -\sqrt{b_{m}^{2}+c_{m}^{2}}, & \text { for } b_{m}>0 \text { or } b_{m}=0, c_{m}<0\end{aligned}\right.$

$a_{k}=x_{l} y_{m}-x_{m} y_{l}$

$b_{k}=y_{l}-y_{m}$

$c_{k}=x_{m}-x_{l}$.

Here $x_{k}, y_{k}$ are the Cartesian coordinates of the corner points 1 to 3 of the triangle, and the subscripts $k, l, m$ always progress modulo 3, i.e., cyclically around the three vertices of the triangle. The shape function vectors for the constant edge elements in Table I are very simple compared with those presented in [18].

Noting that the unit tangential vector on the side between two corner points $\left(x_{k}, y_{k}\right)$ and $\left(x_{l}, y_{l}\right), t_{k}$, is given by

$$
\boldsymbol{t}_{k}=\left(c_{m} / l_{k}\right) \boldsymbol{i}_{x}-\left(b_{m} / l_{k}\right) \boldsymbol{i}_{y}
$$

with $\boldsymbol{i}_{x}, \boldsymbol{i}_{y}$ being the unit vectors in the $x, y$ directions, respectively, it is confirmed from Table I that for the constant edge elements, the following relations are satisfied:

$$
\phi_{t k}=\left(\phi_{x k} \boldsymbol{i}_{x}+\phi_{y k} \boldsymbol{i}_{y}\right) \cdot \boldsymbol{t}_{k}
$$

where $\phi_{x k}, \phi_{y k}(k=1,2,3)$ are the values of $\phi_{x}, \phi_{y}$ at any point on the side of length $\left|l_{k}\right|$, respectively, and thus the tangential component $\phi_{t}$ is constant along each side of the triangle. For the linear edge elements, on the other hand, the following relations are satisfied:

$$
\begin{aligned}
& \phi_{t 1}=\left(\phi_{x 1} \boldsymbol{i}_{x}+\phi_{y 1} \boldsymbol{i}_{y}\right) \cdot \boldsymbol{t}_{1} \\
& \phi_{t 2}=\left(\phi_{x 2} \boldsymbol{i}_{x}+\phi_{y 2} \boldsymbol{i}_{y}\right) \cdot \boldsymbol{t}_{2} \\
& \phi_{t 3}=\left(\phi_{x 3} \boldsymbol{i}_{x}+\phi_{y 3} \boldsymbol{i}_{y}\right) \cdot \boldsymbol{t}_{3} \\
& \phi_{t 4}=\left(\phi_{x 2} \boldsymbol{i}_{x}+\phi_{y 2} \boldsymbol{i}_{y}\right) \cdot \boldsymbol{t}_{1} \\
& \phi_{t 5}=\left(\phi_{x 3} \boldsymbol{i}_{x}+\phi_{y 3} \boldsymbol{i}_{y}\right) \cdot \boldsymbol{t}_{2} \\
& \phi_{t 6}=\left(\phi_{x 1} \boldsymbol{i}_{x}+\phi_{y 1} \boldsymbol{i}_{y}\right) \cdot \boldsymbol{t}_{3}
\end{aligned}
$$

where $\phi_{x k}, \phi_{y k}(k=1,2,3)$ are the values of $\phi_{x}, \phi_{y}$ at the vertex $k$ of the triangle, respectively.
Substituting (7) into (6) and using the same procedure as [18], we obtain the following final eigenvalue problem which gives a solution directly for the propagation constant $\beta$ and the corresponding field distribution and involves only the edge variables in the transverse plane $\left\{\phi_{t}\right\}$ :

$$
\left[K_{t t}\right]\left\{\phi_{t}\right\}-\beta^{2}\left(\left[M_{t t}\right]+\left[K_{t z}\right]\left[K_{z z}\right]^{-1}\left[K_{z t}\right]\right)\left\{\phi_{t}\right\}=\{0\}
$$

with

$$
\begin{aligned}
{\left[K_{t t}\right]=} & \sum_{e} \iint_{e}\left[q_{x} k_{0}^{2}\{U\}\{U\}^{\mathrm{T}}+q_{y} k_{0}^{2}\{V\}\{V\}^{\mathrm{T}}\right. \\
& -p_{z}\left\{U_{y}\right\}\left\{U_{y}\right\}^{\mathrm{T}}-p_{z}\left\{V_{x}\right\}\left\{V_{x}\right\}^{\mathrm{T}} \\
& \left.+p_{z}\left\{U_{y}\right\}\left\{V_{x}\right\}^{\mathrm{T}}+p_{z}\left\{V_{x}\right\}\left\{U_{y}\right\}^{\mathrm{T}}\right] d x d y \\
{\left[K_{t z}\right]=} & {\left[K_{z t}\right]^{\mathrm{T}} } \\
= & \sum_{e} \iint_{e}\left[p_{y}\{U\}\left\{N_{x}\right\}^{\mathrm{T}}+p_{x}\{V\}\left\{N_{y}\right\}^{\mathrm{T}}\right] d x d y
\end{aligned}
$$

$$
\begin{aligned}
{\left[K_{z z}\right]=} & \sum_{e} \iint_{e}\left[q_{z} k_{0}^{2}\{N\}\{N\}^{\mathrm{T}}\right. \\
& \left.-p_{y}\left\{N_{x}\right\}\left\{N_{x}\right\}^{\mathrm{T}}-p_{x}\left\{N_{y}\right\}\left\{N_{y}\right\}^{\mathrm{T}}\right] d x d y \\
{\left[M_{t t}\right]=} & \sum_{e} \iint_{e}\left[p_{y}\{U\}\{U\}^{\mathrm{T}}+p_{x}\{V\}\{V\}^{\mathrm{T}}\right] d x d y
\end{aligned}
$$

where $\{0\}$ is a null vector, $\left\{U_{y}\right\} \equiv \partial\{U\} / \partial y,\left\{V_{x}\right\} \equiv$ $\partial\{V\} / \partial x,\left\{N_{x}\right\} \equiv \partial\{N\} / \partial x,\left\{N_{y}\right\} \equiv \partial\{N\} / \partial y$, and their explicit forms are given in Table II. The integrals necessary to construct element matrices are summarized in the Appendix.

Using (9) to (13) and the Appendix, we can easily construct the matrices $\left[K_{t t}\right],\left[K_{t z}\right],\left[K_{z t}\right],\left[K_{z z}\right]$, and $\left[M_{t t}\right]$.

\section{NUMERICAL RESULTS}

First, in order to check the accuracy of the VFEM with mixed-interpolation-type triangular elements, a half-filled dielectric waveguide as shown in Fig. 2(a) was considered, where $W=2 h$. Fig. 2(b) shows a typical element division profile.

Fig. 3 shows the relative error of the computed $\beta$ for the fundamental $\mathrm{LSE}_{10}$ mode in a rectangular waveguide 


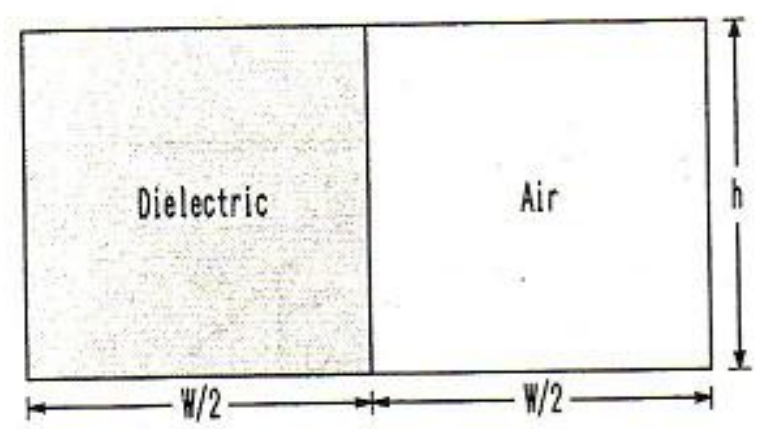

(a)

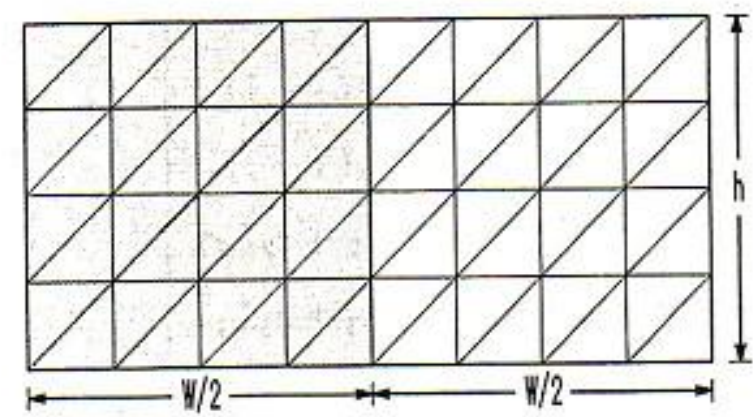

(b)

Fig. 2. Dielectric-loaded waveguide. (a) Waveguide structure. (b) Element division.

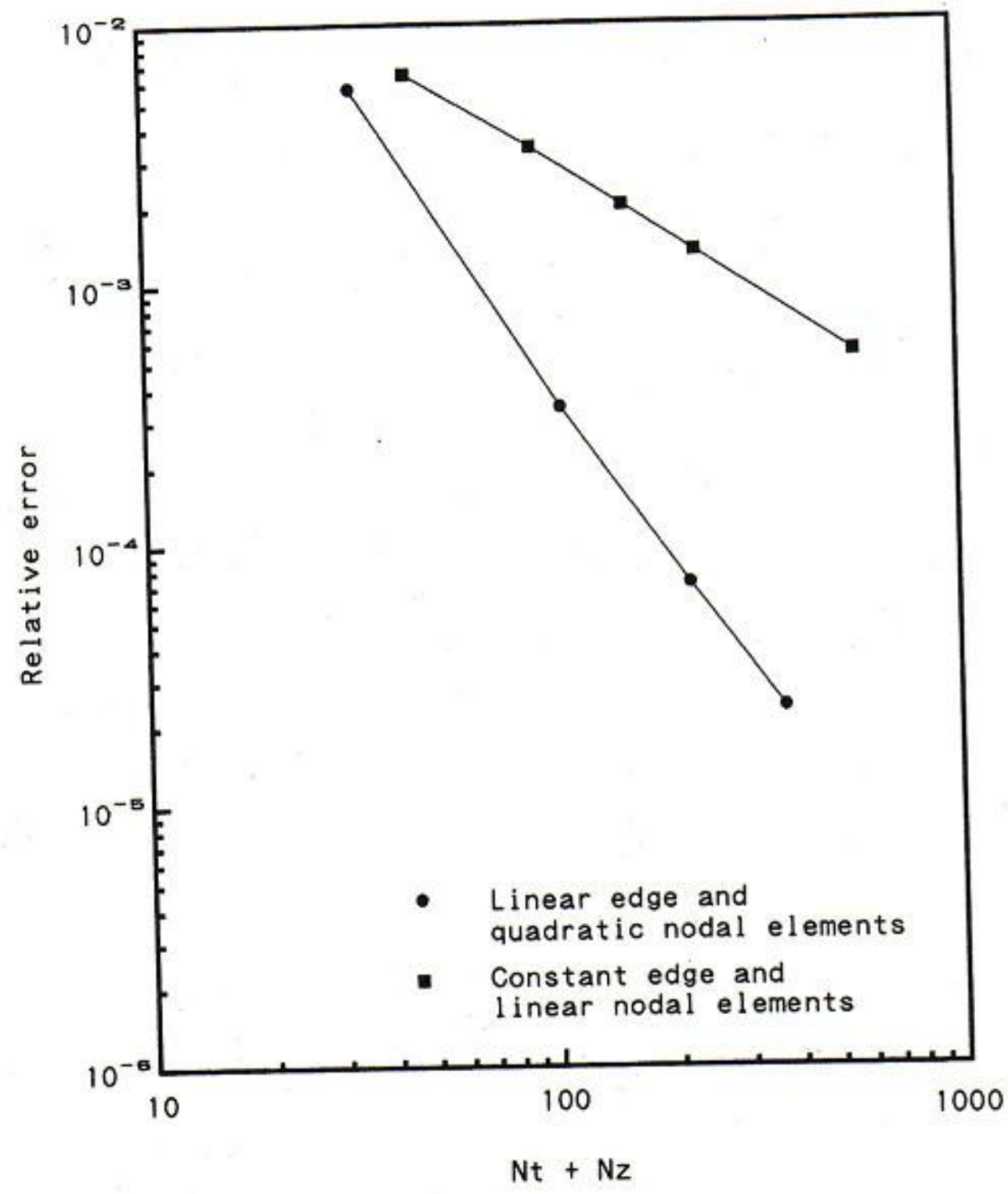

Fig. 3. Convergence of finite element solutions for the fundamental $\mathrm{LSE}_{10}$ mode in a dielectric-loaded waveguide.

inhomogeneously loaded with dielectric of refractive index 1.5 , where $\phi=\boldsymbol{H}, k_{0} h=3.0, N_{t}$ and $N_{z}$ are the numbers of nodes for tangential and axial components, respectively, and $N_{t}+N_{z}$ corresponds to the number of degrees of freedom. The relative error is given by

$$
\text { relative error }=\left(\beta_{\text {exact }}-\beta_{\mathrm{FEM}}\right) / \beta_{\text {exact }}
$$

where $\beta_{\text {exact }}$ and $\beta_{\mathrm{FEM}}$ are the exact and computed values, respectively. It is confirmed from Fig. 3 that the VFEM with the high-order mixed-interpolation-type elements (linear edge and quadratic nodal elements) can give more accurate results

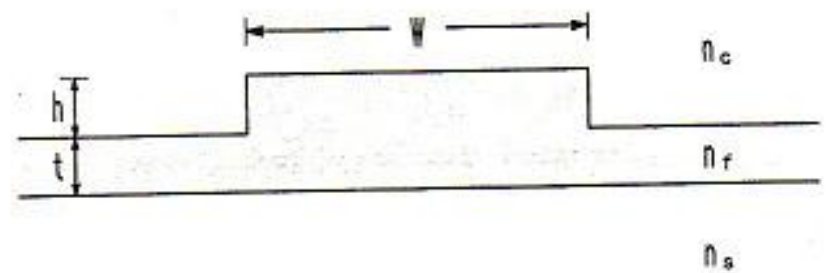

(a)

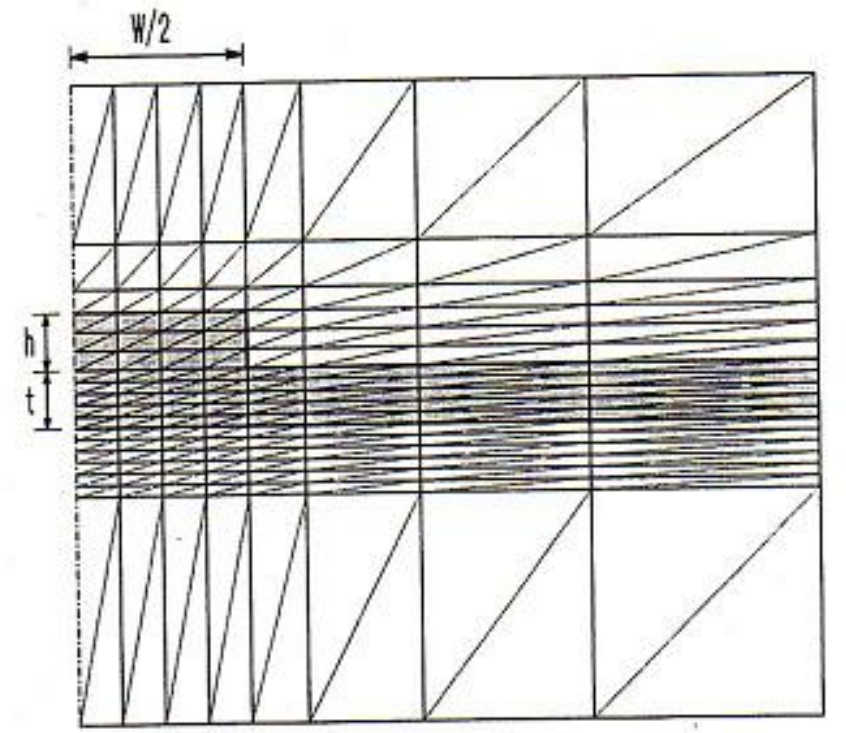

(b)

Fig. 4. Optical rib waveguide. (a) Waveguide structure. (b) Element division.

than the VFEM with the lowest order ones (constant edge and linear nodal elements).

Next, the VFEM with mixed-interpolation-type triangular elements was used to analyze a series of rib waveguides [8], [25]-[27] having, in the notation of Fig. 4(a), rib width $W=3 \mu \mathrm{m}$ and superstrate depth $t+h=1 \mu \mathrm{m}$, where $h$ is the etch depth. The outer slab depth varies from $0 \mu \mathrm{m}$ to $0.9 \mu \mathrm{m}$. The refractive indices of the film, substrate, and cover are $n_{f}=3.44, n_{s}=3.40$, and $n_{c}=1.0$, respectively. The operating wavelength is $1.15 \mu \mathrm{m}$. Fig. 4(b) shows a typical element division profile, where symmetry conditions are used and only one-half of the waveguide cross section is subdivided into linear edge and quadratic nodal elements.

Fig. 5 shows the normalized propagation constant $b$ for the fundamental $E^{x}\left(E_{11}^{x}\right)$ and the fundamental $E^{y}\left(E_{11}^{y}\right)$ modes, where $b$ is defined as

$$
b=\frac{\left(\beta / k_{0}\right)^{2}-n_{s}^{2}}{n_{f}^{2}-n_{s}^{2}}
$$

and $\phi=H$ and $\phi=E$ for the calculation of the $E_{11}^{x}$ and $E_{11}^{y}$ modes, respectively. The results of the VFEM with constant edge and linear nodal elements, the VFEM combined with the penalty function method [8], the effective index method (EIM) [25], the scalar finite difference method (SFDM) [26], and the scalar finite element method (SFEM) [27] are also given in Fig. 5 . When using a VFEM with the high-order or the lowest-order mixed-interpolation-type elements, the number of elements is 288 or 352 , respectively.

The results of the VFEM with the high-order mixedinterpolation-type elements for the $E_{11}^{x}$ mode agree excellently with those of the VFEM combined with the penalty function method [8]. Note that the penalty function method cannot provide a direct solution for the propagation constant and that an extra stage of iteration may be needed if the solution is required at a particular wavelength. The results of the penalty function method have not been reported for the $E_{11}^{y}$ modes. It is readily seen from Fig. 5 that the accuracy of the VFEM 


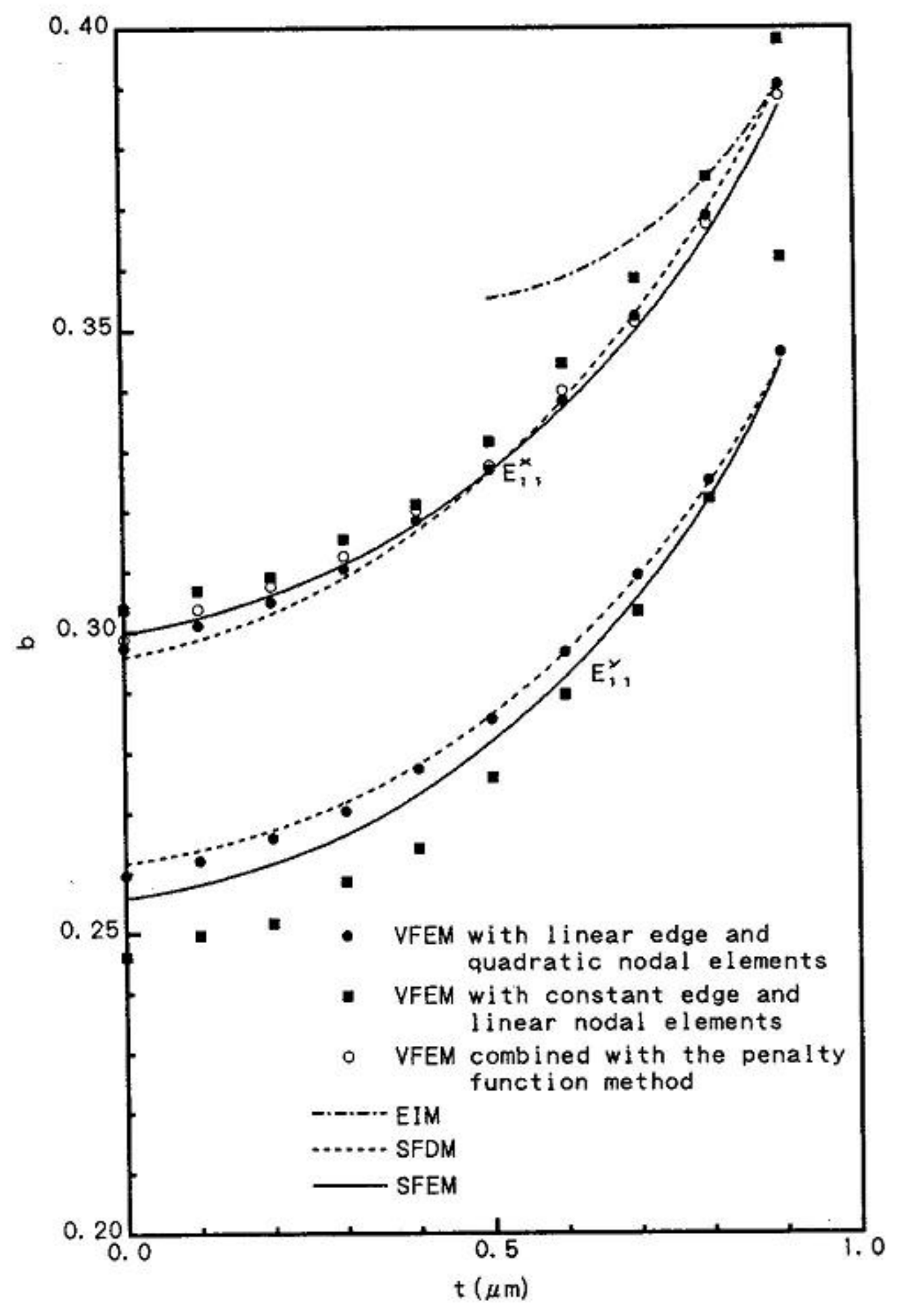

Fig. 5. Normalized propagation constants for the $E_{11}^{x}$ and $E_{11}^{y}$ modes of the rib waveguide.

with the lowest-order mixed-interpolation-type elements is not sufficient. It is also found that for both the $E_{11}^{x}$ and $E_{11}^{y}$ modes, the results of the VFEM with the high-order mixedinterpolation-type elements agree well with those of the SFDM [26] and the SFEM [27]. For detailed comparison the results are summarized in Table III.

Numerical computations for the test problems show the nonappearance of spurious solutions when both the high-order and the lowest-order mixed-interpolation-type elements are used without any other supplementary technique.

\section{CONCLUSION}

A vector finite element method for the analysis of optical waveguiding problems was formulated using the high-order mixed-interpolation-type triangular elements in detail. It is a combination of linear edge elements for transverse components of the electric or magnetic field and quadratic nodal elements for the axial one. This approach can yield more accurate results compared with the conventional approach using the lowest-order mixed-interpolation-type elements, namely, constant edge elements and linear nodal elements. The accuracy of this approach was investigated by calculating the propagation characteristics of optical rib waveguides.
TABLE III

COMparison of Normalized Propagation Constants FOR THE $E_{11}^{x}$ AND $E_{11}^{y}$ MODES OF THE RiB WAVEguIDE

\begin{tabular}{|c|c|c|c|c|c|c|c|}
\hline \multirow{2}{*}{$\begin{array}{c}t \\
(\mu \mathrm{m})\end{array}$} & \multicolumn{4}{|c|}{$E_{11}^{x}$ mode } & \multicolumn{3}{|c|}{$E_{11}^{y}$ mode } \\
\hline & VFEM ${ }^{*}$ & VFEM $^{* *}$ & SFDM & SFEM & VFEM* & SFDM & SFEM \\
\hline 0 & 0.2974 & 0.2988 & 0.2959 & 0.2998 & 0.2596 & 0.2617 & 0.2559 \\
\hline 0.1 & 0.3010 & 0.3038 & 0.2987 & 0.3023 & 0.2621 & 0.2639 & 0.2581 \\
\hline 0.2 & 0.3048 & 0.3075 & 0.3029 & 0.3060 & 0.2659 & 0.2672 & 0.2616 \\
\hline 0.3 & 0.3104 & 0.3125 & 0.3088 & 0.3110 & 0.2703 & 0.2718 & 0.2664 \\
\hline 0.4 & 0.3183 & 0.3200 & 0.3165 & 0.3177 & 0.2773 & 0.2780 & 0.2731 \\
\hline 0.5 & 0.3268 & 0.3275 & 0.3263 & 0.3265 & 0.2856 & 0.2862 & 0.2818 \\
\hline 0.6 & 0.3382 & 0.3399 & 0.3382 & 0.3369 & 0.2965 & 0.2964 & 0.2923 \\
\hline 0.7 & 0.3522 & 0.3512 & 0.3525 & 0.3497 & 0.3093 & 0.3089 & 0.3055 \\
\hline 0.8 & 0.3687 & 0.3674 & 0.3696 & 0.3656 & 0.3250 & 0.3245 & 0.3220 \\
\hline 0.9 & 0.3905 & 0.3886 & 0.3905 & 0.3869 & 0.3462 & 0.3448 & 0.3444 \\
\hline
\end{tabular}

VFEM* : VFEM with the high-order mixed-interpolation-type elements VFEM** : VFEM combined with the penalty function method

This approach can be applied easily to the optical waveguides including lossy and/or active media.

\section{APPENDIX}

The integrals necessary to construct element matrices are calculated as follows.

Constant Edge and Linear Nodal Elements:

$$
\begin{gathered}
{\left[\iint_{e}\{U\}\{U\}^{\mathrm{T}} d x d y\right]_{i j}} \\
=\frac{1}{4 A_{e}} l_{i} l_{j}\left[y_{i+2} y_{j+2}-y_{c}\left(y_{i+2}+y_{j+2}\right)\right. \\
\left.\quad+\frac{1}{12}\left(y_{1}^{2}+y_{2}^{2}+y_{3}^{2}+9 y_{c}^{2}\right)\right] \\
{\left[\int_{e}\{V\}\{V\}^{\mathrm{T}} d x d y\right]_{i j}} \\
=\frac{1}{4 A_{e}} l_{i} l_{j}\left[x_{i+2} x_{j+2}-x_{c}\left(x_{i+2}+x_{j+2}\right)\right. \\
\left.+\frac{1}{12}\left(x_{1}^{2}+x_{2}^{2}+x_{3}^{2}+9 x_{c}^{2}\right)\right]
\end{gathered}
$$

$$
\begin{aligned}
{\left[\iint_{e}\left\{U_{y}\right\}\left\{U_{y}\right\}^{\mathrm{T}} d x d y\right]_{i j} } & =\left[\iint_{e}\left\{V_{x}\right\}\left\{V_{x}\right\}^{\mathrm{T}} d x d y\right]_{i j} \\
& =-\left[\iint_{e}\left\{U_{y}\right\}\left\{V_{x}\right\}^{\mathrm{T}} d x d y\right]_{i j} \\
& =-\left[\iint_{e}\left\{V_{x}\right\}\left\{U_{y}\right\}^{\mathrm{T}} d x d y\right]_{i j} \\
& =\frac{1}{4 A_{e}} l_{i} l_{j}
\end{aligned}
$$




$$
\begin{aligned}
& {\left[\iint_{e}\{U\}\left\{N_{x}\right\}^{\mathrm{T}} d x d y\right]_{i j}=\frac{1}{4 A_{e}} l_{i} b_{j}\left(y_{i+2}-y_{c}\right)} \\
& {\left[\iint_{e}\{V\}\left\{N_{y}\right\}^{\mathrm{T}} d x d y\right]_{i j}=\frac{1}{4 A_{e}} l_{i} c_{j}\left(x_{c}-x_{i+2}\right)} \\
& {\left[\iint_{e}\{N\}\{N\}^{\mathrm{T}} d x d y\right]_{i j}= \begin{cases}A_{e} / 6, & \text { for } i=j \\
A_{e} / 12, & \text { for } i \neq j\end{cases} } \\
& {\left[\iint_{e}\left\{N_{x}\right\}\left\{N_{x}\right\}^{\mathrm{T}} d x d y\right]_{i j}=\frac{1}{4 A_{e}} b_{i} b_{j}} \\
& {\left[\iint_{e}\left\{N_{y}\right\}\left\{N_{y}\right\}^{\mathrm{T}} d x d y\right]_{i j}=\frac{1}{4 A_{e}} c_{i} c_{j}}
\end{aligned}
$$

with

$$
\begin{aligned}
& x_{c}=\left(x_{1}+x_{2}+x_{3}\right) / 3 \\
& y_{c}=\left(y_{1}+y_{2}+y_{3}\right) / 3
\end{aligned}
$$

where $[\cdot]_{i j}(i j=11,12, \cdots, 33)$ indicates the $(i, j)$ component of the matrix [.] , and the subscripts $i, j$ always progress modulo 3.

\section{Linear Edge and Quadratic Nodal Elements:}

$$
\begin{aligned}
& {\left[\iint_{e}\{U\}\{U\}^{\mathrm{T}} d x d y\right]_{i j}} \\
& = \begin{cases}\frac{A_{e}}{6} u_{i} u_{j} & \text { for } i j=11,22,33,44,55,66 \\
\frac{A_{e}}{12} u_{i} u_{j}, & \text { for others }\end{cases} \\
& {\left[\iint_{e}\{V\}\{V\}^{\mathrm{T}} d x d y\right]_{i j}} \\
& = \begin{cases}\frac{A_{e}}{6} v_{i} v_{j} & \text { for } i j=11,22,33,44,55,66, \\
\frac{A_{e}}{12} v_{i} v_{j}, & \text { for others }\end{cases}
\end{aligned}
$$

$\left[\iint_{e}\left\{U_{y}\right\}\left\{U_{y}\right\}^{\mathrm{T}} d x d y\right]_{i j}=A_{e} u_{y i} u_{y j}$

$\left[\iint_{e}\left\{V_{x}\right\}\left\{V_{x}\right\}^{\mathrm{T}} d x d y\right]_{i j}=A_{e} v_{x i} v_{x j}$

$$
\left[\iint_{e}\left\{U_{y}\right\}\left\{V_{x}\right\}^{\mathrm{T}} d x d y\right]_{i j}=A_{e} u_{y i} v_{x j}
$$

$$
\begin{aligned}
& {\left[\iint_{e}\left\{V_{x}\right\}\left\{U_{y}\right\}^{\mathrm{T}} d x d y\right]_{i j}=A_{e} v_{x i} u_{y j}} \\
& {\left[\iint_{e}\{U\}\left\{N_{x}\right\}^{\mathrm{T}} d x d y\right]_{1 j}=\frac{A_{e}}{12} u_{1}\left(2 C_{x j}^{(1)}+C_{x j}^{(2)}\right.} \\
& \left.+C_{x j}^{(3)}+4 C_{x j}^{(4)}\right) \\
& {\left[\iint_{e}\{U\}\left\{N_{x}\right\}^{\mathrm{T}} d x d y\right]_{2 j}=\frac{A_{e}}{12} u_{2}\left(C_{x j}^{(1)}+2 C_{x j}^{(2)}\right.} \\
& \left.+C_{x j}^{(3)}+4 C_{x j}^{(4)}\right) \\
& {\left[\iint_{e}\{U\}\left\{N_{x}\right\}^{\mathrm{T}} d x d y\right]_{3 j}=\frac{A_{e}}{12} u_{3}\left(C_{x j}^{(1)}+C_{x j}^{(2)}\right.} \\
& \left.+2 C_{x j}^{(3)}+4 C_{x j}^{(4)}\right)
\end{aligned}
$$

$\left[\iint_{e}\{U\}\left\{N_{x}\right\}^{\mathrm{T}} d x d y\right]_{4 j}=\frac{A_{e}}{12} u_{4}\left(C_{x j}^{(1)}+2 C_{x j}^{(2)}\right.$

$$
\left.+C_{x j}^{(3)}+4 C_{x j}^{(4)}\right)
$$

$\left[\iint_{e}\{U\}\left\{N_{x}\right\}^{\mathrm{T}} d x d y\right]_{5 j}=\frac{A_{e}}{12} u_{5}\left(C_{x j}^{(1)}+C_{x j}^{(2)}\right.$

$$
\left.+2 C_{x j}^{(3)}+4 C_{x j}^{(4)}\right)
$$

$\left[\iint_{e}\{U\}\left\{N_{x}\right\}^{\mathrm{T}} d x d y\right]_{6 j}=\frac{A_{e}}{12} u_{6}\left(2 C_{x j}^{(1)}+C_{x j}^{(2)}\right.$

$$
\left.+C_{x j}^{(3)}+4 C_{x j}^{(4)}\right)
$$

$\left[\iint_{e}\{V\}\left\{N_{y}\right\}^{\mathrm{T}} d x d y\right]_{1 j}=\frac{A_{e}}{12} v_{1}\left(2 C_{y j}^{(1)}+C_{y j}^{(2)}\right.$

$$
\left.+C_{y j}^{(3)}+4 C_{y j}^{(4)}\right)
$$

$\left[\iint_{e}\{V\}\left\{N_{y}\right\}^{\mathrm{T}} d x d y\right]_{2 j}=\frac{A_{e}}{12} v_{2}\left(C_{y j}^{(1)}+2 C_{y j}^{(2)}\right.$

$$
\left.+C_{y j}^{(3)}+4 C_{y j}^{(4)}\right)
$$

$\left[\iint_{e}\{V\}\left\{N_{y}\right\}^{\mathrm{T}} d x d y\right]_{3 j}=\frac{A_{e}}{12} v_{3}\left(C_{y j}^{(1)}+C_{y j}^{(2)}\right.$

$$
\left.+2 C_{y j}^{(3)}+4 C_{y j}^{(4)}\right)
$$

$$
\begin{aligned}
{\left[\iint_{e}\{V\}\left\{N_{y}\right\}^{\mathrm{T}} d x d y\right]_{4 j}=} & \frac{A_{e}}{12} v_{4}\left(C_{y j}^{(1)}+2 C_{y j}^{(2)}\right. \\
& \left.+C_{y j}^{(3)}+4 C_{y j}^{(4)}\right)
\end{aligned}
$$

$\left[\iint_{e}\{V\}\left\{N_{y}\right\}^{\mathrm{T}} d x d y\right]_{5 j}=\frac{A_{e}}{12} v_{5}\left(C_{y j}^{(1)}+C_{y j}^{(2)}\right.$

$$
\left.+2 C_{y j}^{(3)}+4 C_{y j}^{(4)}\right)
$$


TABLE IV

VALUES OF $u_{i}, v_{i}, U_{y i}, v_{x i}$, AND $C_{x i}^{(1)}$ To $C_{y i}^{(4)}$

\begin{tabular}{ccccccccccccc}
\hline \hline$i$ & $u_{i}$ & $v_{i}$ & $u_{y i}$ & $v_{x i}$ & $C_{x i}^{(1)}$ & $C_{x i}^{(2)}$ & $C_{x i}^{(3)}$ & $C_{x i}^{(4)}$ & $C_{y i}^{(1)}$ & $C_{y i}^{(2)}$ & $C_{y i}^{(3)}$ & $C_{y i}^{(4)}$ \\
\hline 1 & $l_{1} b_{2}$ & $l_{1} c_{2}$ & $u_{1} c_{1}$ & $v_{1} b_{1}$ & $4 b_{1}$ & 0 & 0 & $-b_{1}$ & $4 c_{1}$ & 0 & 0 & $-c_{1}$ \\
2 & $l_{2} b_{3}$ & $l_{2} c_{3}$ & $u_{2} c_{2}$ & $v_{2} b_{2}$ & 0 & $4 b_{2}$ & 0 & $-b_{2}$ & 0 & $4 c_{2}$ & 0 & $-c_{2}$ \\
3 & $l_{3} b_{1}$ & $l_{3} c_{1}$ & $u_{3} c_{3}$ & $v_{3} b_{3}$ & 0 & 0 & $4 b_{3}$ & $-b_{3}$ & 0 & 0 & $4 c_{3}$ & $-c_{3}$ \\
4 & $-l_{1} b_{1}$ & $-l_{1} c_{1}$ & $u_{4} c_{2}$ & $v_{4} b_{2}$ & $4 b_{2}$ & $4 b_{1}$ & 0 & 0 & $4 c_{2}$ & $4 c_{1}$ & 0 & 0 \\
5 & $-l_{2} b_{2}$ & $-l_{2} c_{2}$ & $u_{5} c_{3}$ & $v_{5} b_{3}$ & 0 & $4 b_{3}$ & $4 b_{2}$ & 0 & 0 & $4 c_{3}$ & $4 c_{2}$ & 0 \\
6 & $-l_{3} b_{3}$ & $-l_{3} c_{3}$ & $u_{6} c_{1}$ & $v_{6} b_{1}$ & $4 b_{3}$ & 0 & $4 b_{1}$ & 0 & $4 c_{3}$ & 0 & $4 c_{1}$ & 0 \\
\hline
\end{tabular}

Common denominator : $1 / 2 A_{e}$

$$
\begin{aligned}
{\left[\iint_{e}\{V\}\left\{N_{y}\right\}^{\mathrm{T}} d x d y\right]_{6 j}=} & \frac{A_{e}}{12} v_{6}\left(2 C_{y j}^{(1)}+C_{y j}^{(2)}\right. \\
& \left.+C_{y j}^{(3)}+4 C_{y j}^{(4)}\right)
\end{aligned}
$$

$$
\begin{aligned}
& \iint_{e}\{N\}\{N\}^{\mathrm{T}} d x d y \\
& =\frac{A_{e}}{180}\left[\begin{array}{rrrrrr}
6 & -1 & -1 & 0 & -4 & 0 \\
-1 & 6 & -1 & 0 & 0 & -4 \\
-1 & -1 & 6 & -4 & 0 & 0 \\
0 & 0 & -4 & 32 & 16 & 16 \\
-4 & 0 & 0 & 16 & 32 & 16 \\
0 & -4 & 0 & 16 & 16 & 32
\end{array}\right]
\end{aligned}
$$$$
\left[\iint_{e}\left\{N_{x}\right\}\left\{N_{x}\right\}^{\mathrm{T}} d x d y\right]_{i j}
$$$$
=\frac{A_{e}}{6}\left(C_{x i}^{(1)} C_{x j}^{(1)}+C_{x i}^{(2)} C_{x j}^{(2)}+C_{x i}^{(3)} C_{x j}^{(3)}\right)
$$$$
+\frac{A_{e}}{12}\left(C_{x i}^{(1)} C_{x j}^{(2)}+C_{x i}^{(1)} C_{x j}^{(3)}+C_{x i}^{(2)} C_{x j}^{(1)}\right.
$$$$
\left.+C_{x i}^{(2)} C_{x j}^{(3)}+C_{x i}^{(3)} C_{x j}^{(1)}+C_{x i}^{(3)} C_{x j}^{(2)}\right)
$$$$
+\frac{A_{e}}{3}\left(C_{x i}^{(1)} C_{x j}^{(4)}+C_{x i}^{(2)} C_{x j}^{(4)}+C_{x i}^{(3)} C_{x j}^{(4)}\right.
$$$$
\left.+C_{x i}^{(4)} C_{x j}^{(1)}+C_{x i}^{(4)} C_{x j}^{(2)}+C_{x i}^{(4)} C_{x j}^{(3)}\right)
$$$$
+A_{e} C_{x i}^{(4)} C_{x j}^{(4)}
$$

$$
\begin{aligned}
& {\left[\int_{e}\left\{N_{y}\right\}\left\{N_{y}\right\}^{\mathrm{T}} d x d y\right]_{i j} } \\
&=\frac{A_{e}}{6}\left(C_{y i}^{(1)} C_{y j}^{(1)}+C_{y i}^{(2)} C_{y j}^{(2)}+C_{y i}^{(3)} C_{y j}^{(3)}\right) \\
&+\frac{A_{e}}{12}\left(C_{y i}^{(1)} C_{y j}^{(2)}+C_{y i}^{(1)} C_{y j}^{(3)}+C_{y i}^{(2)} C_{y j}^{(1)}\right. \\
&\left.+C_{y i}^{(2)} C_{y j}^{(3)}+C_{y i}^{(3)} C_{y j}^{(1)}+C_{y i}^{(3)} C_{y j}^{(2)}\right) \\
&+\frac{A_{e}}{3}\left(C_{y i}^{(1)} C_{y j}^{(4)}+C_{y i}^{(2)} C_{y j}^{(4)}+C_{y i}^{(3)} C_{y j}^{(4)}\right. \\
&\left.+C_{y i}^{(4)} C_{y j}^{(1)}+C_{y i}^{(4)} C_{y j}^{(2)}+C_{y i}^{(4)} C_{y j}^{(3)}\right) \\
&+A_{e} C_{y i}^{(4)} C_{y j}^{(4)}
\end{aligned}
$$

where $[\cdot]_{i j}(i j=11,12, \cdots, 66)$ indicates the $(i, j)$ component of the matrix [·], and the values of $u_{i}, v_{i}, u_{y i}, v_{x i}$, and $C_{x i}^{(1)}$ to $C_{y i}^{(4)}$ are listed in Table IV.

\section{ACKNOWLEDGMENT}

The authors are greatly indebted to the reviewers for their helpful suggestions and for pointing out [15], [16], and [22]-[24].

\section{REFERENCES}

[1] M. Koshiba, K. Hayata, and M. Suzuki, "Vectorial finite-element formulation without spurious modes for dielectric waveguides," Trans. Inst. Electron. Commun. Eng. Japan, vol. E67, pp. 191-196, Apr. 1984.

[2] —, "Vectorial finite-element formulation without spurious solutions for dielectric waveguide problems," Electron. Lett., vol. 20, pp. 409-410, May 1984.

[3] B. M. A. Rahman and J. B. Davies, "Penalty function improvement of waveguide solution by finite elements," IEEE Trans. Microwave Theory Tech., vol. MTT-32, pp. 922-928, Aug. 1984.

[4] J. B. Davies and B. M. A. Rahman, "Analysis of open optical and microwave guides of arbitrary transverse permittivity tensor," Radio Sci., vol. 19, pp. 1245-1249, Sept.-Oct. 1984.

[5] B. M. A. Rahman and J. B. Davies, "Finite-element solution of integrated optical waveguides," J. Lightwave Technol, vol. LT-2, pp. 682-688, Oct. 1984.

[6] M. Koshiba, K. Hayata, and M. Suzuki, "Improved finite-element formulation in terms of magnetic field vector for dielectric waveguides," IEEE Trans. Microwave Theory Tech., vol. MTT-33, pp. 227-233, Mar. 1985.

[7] —, "Finite-element formulation in terms of the electric-field vector for electromagnetic waveguide problems," IEEE Trans. Microwave Theory Tech., vol. MTT-33, pp. 900-906, Oct. 1985.

[8] B. M. A. Rahman and J. B. Davies, "Vector- $H$ finite element solution of GaAs/GaAlAs rib waveguides," IEE Proc., pt. J, vol. 132, pp. 349-353, Dec. 1985 .

[9] M. Koshiba, K. Hayata, and M. Suzuki, "Vector $E$-field finite-element analysis of dielectric optical waveguides," Appl. Opt., vol. 25, pp. 10-11. Jan. 1986.

[10] - "Finite-element solution of anisotropic waveguides with arbitrary tensor permittivity," J. Lightwave Technol., vol. LT-4, pp. 121-126, Feb. 1986.

[11] K. Hayata, M. Koshiba, and M. Suzuki, "Vertorial wave analysis of stress-applied polarization-maintaining optical fibers by the finiteelement method," J. Lightwave Technol, vol. LT-4, pp. 133-139, Feb. 1986.

[12] —- "Lateral mode analysis of buried heterostructure diode lasers by the finite-element method," IEEE J. Quantum Electron., vol. QE-22, pp. 781-788, June 1986.

[13] K. Hayata, M. Eguchi, M. Koshiba, and M. Suzuki, "Vectorial wave analysis of side-tunnel type polarization-maintaining optical fibers by variational finite elements," J. Lightwave Technol., vol. LT-4, pp. 1090-1096, Aug. 1986.

[14] T. P. Young, "Design of integrated optical circuits using finite elements," IEE Proc., pt. A, vol, 135, pp. 135-144, Mar. 1988.

[15] M. Sh. Birman, "The Maxwell operator for a resonator with inward edges," Vestnik Leningradskogo Universiteta. Matematika, vol. 19, pp. $1-8,1986$.

[16] M. Sh. Birman and M. Z. Solomyak, "Maxwell operator in regions with nonsmooth boundaries," Siberian Math. J., vol. 28, pp. 12-24, 1987.

[17] F. Kikuchi, "Mixed and penalty formulations for finite element analysis of an eigenvalue problem in electromagnetism," Comput. Methods Appl. Mech. Eng., vol. 64, pp. 509-521, 1987.

[18] M. Koshiba and K. Inoue, "Simple and efficient finite-element analysis of microwave and optical waveguides," IEEE Trans. Microwave Theory Tech., vol. 40, pp. 371-377, Feb. 1992.

[19] A. Bermúdez and D. G. Pedreira, "Mathematical analysis of a finite element method without spurious solutions for computation of dielectric waveguides," Numer. Math., vol. 61, pp. 39-57, 1992.

[20] M. Hano, "Vector finite-element solution of anisotropic waveguides using novel triangular elements," Electron. Commun. Japan, pt. 2, vol. 71, pp. 71-80, 1988.

[21] J.-F. Lee, D.-K. Sun, and Z. J. Cendez, "Full-wave analysis of dielectric waveguides using tangential vector finite elements," IEEE Trans. Microwave Theory Tech., vol. 39, pp. 1262-1271, Aug. 1991. 
[22] F. Brezzi, J. Douglas, Jr., and L. D. Marini, "Two families of mixed finite elements for second order elliptic problems," Numer. Math., vol. 47, pp. 217-235, 1985.

[23] R. G. Durán, "Finite elements in $H$ (curl) with applications to elasticity," Numer. Methods Partial Differential Equations, vol. 4, pp. 167-175, 1990.

[24] J. C. Nédélec, "A new family of mixed elements in $R^{3}$, Numer. Math., vol. 50 , pp. 57-81, 1986.

[25] P. C. Kendal, M. J. Adams, S. Ritchie, and M. J. Robertson, "Theory for calculating approximate values for the propagation constants of an optical rib waveguide by weighting the refractive indices," IEE Proc., pt. J, vol. 134, pp. 699-702, Sept. 1987.

[26] M. S. Stern, "Semivectorial polarized finite difference method for optical waveguides with arbitrary index profiles," IEE Proc., pt. J, vol. 135, pp. 56-63, Feb. 1988.

[27] M. Koshiba, H. Saitoh, M. Eguchi, and K. Hirayama, "Simple scalar finite element approach to optical rib waveguides," IEE Proc., pt. J, vol. 139, pp. 166-171, Apr. 1992.
Manasori Koshiba, photograph and biography not available at the time of publication.

Shinji Maruyama, photograph and biography not available at the time of publication.

Koichi Hirayama, photograph and biography not available at the time of publication. 